\title{
A 6-month randomised controlled trial investigating effects of Mediterranean-style diet and fish oil supplementation on dietary behaviour change, mental and cardiometabolic health and health-related quality of life in adults with depression (HELFIMED): study protocol
}

Dorota Zarnowiecki ${ }^{1}$, Jihyun Cho ${ }^{1}$, Amy Wilson ${ }^{3}$, Svetlana Bogomolova ${ }^{3}$, Anthony Villani ${ }^{2}$, Catherine Itsiopoulos ${ }^{4}$, Theo Niyonsenga ${ }^{1}$, Kerin O'Dea', Sarah Blunden ${ }^{5}$, Barbara Meyer ${ }^{6}$, Leonie Segal ${ }^{1}$ and Natalie Parletta ${ }^{1,7^{*}}$ (D)

\begin{abstract}
Background: Modern diets, characterised by excess consumption of processed foods, are accompanied by an epidemic of chronic diseases. Cardiovascular disease and depression carry a large burden of disease and often occur together. Poor dietary patterns have been identified as an independent risk factor for depression while healthy diets with minimally processed food are protective. Traditional Mediterranean diets have been shown to reduce risk of cardiovascular disease; however there is a need for clinical trials in people with depression. This paper reports the study protocol of a Mediterranean-style diet intervention conducted in adults with self-reported depression.

Methods/design: HELFIMED is a parallel 6-month randomised controlled trial investigating whether dietary patterns can be improved in people with depressive symptoms and whether healthier diet combined with fish oil supplementation can improve mental and cardiometabolic health and quality of life. Adults aged 18-65 years with selfreported depressive symptoms over the previous two months $(N=163)$ were block-randomised on age and gender between May 2014 and June 2015 to receive nutrition education, food hampers, fortnightly cooking workshops based on Mediterranean-style dietary principles for 3 months and fish oil supplementation for 6 months, or to attend fortnightly social groups (control group) for 3 months. All participants completed mental health (DASS and PANAS), quality of life, dietary and shopping and budgeting questionnaires and provided anthropometric measurements, blood and urine samples at baseline, 3 and 6 months. Additionally the treatment group attended focus groups at 3 and 6 months. Primary and secondary outcomes will be analysed using linear mixed modelling and correlations will investigate associations between improved health outcomes and subjective/objective measures of improved diet/ nutritional status.

(Continued on next page)
\end{abstract}

\footnotetext{
* Correspondence: natalie.parletta@unisa.edu.au

${ }^{1}$ Centre for Population Health Research, Sansom Institute for Health Research,

School of Health Sciences, University of South Australia, Adelaide, Australia

${ }^{7}$ Centre for Population Health Research, School of Health Sciences, University

of South Australia, GPO Box 2471, Adelaide, SA 5001, Australia

Full list of author information is available at the end of the article
} 
(Continued from previous page)

Discussion: This study will contribute causal evidence to prior observational and longitudinal studies that have shown associations between Mediterranean diet and mental health. The results will inform public health and clinical strategies for treatment of depression and comorbid cardiometabolic risk factors.

Trial registration: Australian New Zealand Clinical Trials Register (ACTRN12614000438651). Trial registration date: $23^{\text {rd }}$ April 2014.

Keywords: Mediterranean diet, Depression, Cardiometabolic health, Quality of life, Study protocol

Abbreviations: ADHD, Attention deficit hyperactivity disorder; apoA1, Apolipoprotein A1; apoB, Apolipoprotein B; AQoL, Assessment of quality of life; DASS, Depression anxiety stress scale; DHA, Docosahexaenoic acid;

EPA, Eicosapentaenoic acid; HELFIMED, Healthy eating for life with a MEDiterranean-style diet; n-3

PUFAs, Omega-3 polyunsaturated fatty acids; PANAS, Positive and negative affect scale; SDQ, Simple dietary

questionnaire

\section{Background}

Unhealthy food consumption has worsened around the globe, including developed countries, as evidenced by a recent systematic assessment of dietary quality in 187 countries from 1990-2010 [1]. Diet-related health burdens have resulted in a major global shift towards an epidemic of non-communicable chronic disease [2]. Despite advances in drug treatments, the World Health Organization predicted that mental disorders, specifically depression, and heart disease will be the second and third leading causes of disability and will be major contributors to the global burden of disease by 2030 [3]. Moreover there is evidence that physical health conditions, such as cardiovascular disease and metabolic syndrome, and mental illness including depression share similar underlying mechanisms such as chronic inflammation [4-7].

Mediterranean-style diets, characterised by high consumption of vegetables, fruit, legumes, olive oil, fish, cereals, nuts and seeds, moderate consumption of red wine with meals, and low intakes of processed food, red meat and vegetable oils, are rich in bio-protective nutrients, fibre, healthy fats, antioxidants and polyphenolic compounds $[8,9]$. Accordingly, these diets have been associated with improved cardiovascular health and decreased mortality, with protective benefits for cancer, obesity, cardiovascular disease and diabetes $[10,11]$, and this has been supported by intervention studies [12, 13]. Mechanisms of action that have been identified via metaanalysis of studies comparing the Mediterranean diet with low-fat diet in overweight individuals at increased risk of cardiovascular disease include reduced total and LDL cholesterol, increased HDL cholesterol, and reduced inflammation, fasting glucose and insulin [13].

In comparison to focus on physical health problems, relatively little attention has been given to the implications of detrimental modern dietary patterns for society's growing mental health problems, despite the important structural and functional roles of nutrients in the brain [14].
Nutritional supplement interventions have shown some promise in improving cognitive function and mental health. In particular, considerable research has in recent decades investigated benefits of omega-3 polyunsaturated fatty acids (n-3 PUFAs) for mental health across the lifespan including attention deficit hyperactivity disorder (ADHD) and learning difficulties, uni- and bipolar depression, schizophrenia and mild cognitive impairment $[15,16]$. However, a range of nutrients and other food components (e.g. polyphenols, fibre) provided by a whole food, Mediterranean-style diet are important for physical and mental health. There is increasing evidence that diet not only plays a crucial role in gut health but also subsequently on brain function via the gut-brain axis $[14,15]$. While there is converging evidence showing an important role for individual nutrients and dietary components in brain function, there is a dearth of research investigating synergistic actions of nutrients and benefits of whole diets for mental health [14].

Correlational and longitudinal population studies have reported that healthy dietary patterns are associated with lower incidence of depression while unhealthy dietary patterns are associated with higher risk [17]. The Mediterranean diet has been associated in case-control, longitudinal cohort and cross-sectional studies with reduced risk of depression [18]. However a very limited number of dietary interventions has investigated mental health benefits with urgent calls for more research in this area $[19,20]$. The PREDIMED study recruited adults with high risk for cardiovascular disease and allocated participants to one of two Mediterranean diet groups supplemented with nuts or olive oil or a control group that was allocated to a low-fat diet [21]. Secondary analysis at 3-year follow-up identified that incidence of depression was inversely associated with the Mediterranean diet plus nuts group although non-significantly. This finding became statistically significant when restricted to participants with Type 2 diabetes mellitus, showing a $41 \%$ relative reduction in depression risk. Further per 
protocol analysis revealed that strongest adherence to Mediterranean diet was associated with lowest depression risk [22]. In a weight-loss intervention, improvements in diet quality over six months in both the Mediterranean diet intervention group and control diet based on American Heart Association guidelines were associated with over 50 \% reduction in Beck Depression Inventory (BDI) scores [23]. Additional longitudinal analyses adjusting for confounders showed improvements in BDI scores to be mediated by higher fibre, folate and magnesium consumption, however only folate remained as a significant mediator after adjustment for weight-loss [23]. Stahl et al. recruited older adults at risk for major depression to investigate the efficacy of problem solving for reducing major depressive episodes and used a dietary coaching arm, based on US dietary guidelines, as a control [24]. Contrary to their expectations, they found that the dietary arm decreased depressive symptoms by 40-50\%, which was maintained over 2 year follow-up, and that the rate of progression to major depression was greatly reduced compared to population norms. Interestingly, medical comorbidities were one of the predictors of transition to major depression. Based on this evidence there is a need for randomised controlled trials to recruit people with depression to investigate whether dietary intervention, with a focus on Mediterranean-style diet, can reduce depressive symptoms and improve wellbeing.

This study aims to investigate the effects of a 6-month Mediterranean-style dietary intervention combined with fish oil supplementation on mental health, cardiometabolic health and health-related quality of life in people with selfreported depression. The current paper provides a comprehensive outline of the study protocol and measures used. To our knowledge this will be one of the first randomised controlled trials worldwide to investigate the benefits of Mediterranean-style diet on mental health in people with self-reported depression, with one other Australian study underway [25]. These studies will help to address an important gap in the evidence base for addressing the growing global public health challenges associated with depression and cardiovascular disease.

\section{Method/design}

\section{Aims}

The aims of the study, "Healthy Eating for LiFe with a MEDiterranean-style diet" (HELFIMED) are to (a) encourage participants to adopt a healthy whole food, Mediterranean-style diet via provision of cooking workshops supplemented by recipe books and cooking videos, food hampers and nutrition education; and (b) measure benefits of improved diet and fish oil supplementation for cardiometabolic and mental health and health-related quality of life. Secondary aims are to investigate whether (a) improved self-reported diet and dietary biomarkers (carotenoids, urinary sodium and potassium), and erythrocyte (red blood cell) n-3 PUFA levels from fish oil supplementation are associated with improved mental and/or cardiometabolic health outcomes; and (b) outcomes are mediated by inflammatory and oxidative markers. Depression is a risk factor for developing cardiovascular disease, and furthermore, increases mortality from cardiovascular disease [26]. This may be attributed to common risk factors including poor diet and low n-3 PUFA levels which are associated with both conditions. Given the alarmingly low n-3 PUFA intake in western diets [27] and their importance for cardiovascular and mental health, fish oil supplementation was included in this study. Indeed baseline analysis from this cohort and another cohort of people with schizophrenia identified an average omega-3 index (eicosapentaenoic acid; EPA + docosahexaenoic acid; DHA) [28] of $3.95(\mathrm{SD}=1.06)$ percent of red blood cell membranes [29] which is in the range that confers highest risk of mortality from cardiovascular disease $(\leq 4 \%)$, and is even lower than the average low level of $5 \%$ in the Australian population [30,31].

\section{Participants and study procedure}

Adults aged 18-65 years $(N=163)$ with self-reported depression, shown to have a moderate level of agreement with diagnosed depression [32], were recruited from the community via newspaper advertisements, television and radio interviews, social media, and a market research agency for a 6-month parallel randomised controlled intervention with data collected at 0,3 and 6 months. Ethics approval was provided by the Human Research Ethics Committee of the University of South Australia (registration no. 0000032674) and all participants provided written informed consent. According to Cohen's power analyses, at least 60 people in each group are required to detect a medium effect size with $80 \%$ power; therefore allowing for an estimated $30 \%$ dropout rate the target total sample size was $N=172$. A CONSORT flow chart of participants through the study is provided in Fig. 1.

Interested volunteers were screened via phone for the following eligibility criteria: 18-65 years old; diagnosed depression or self-reported depressive symptoms over the past 2 months or more; not taking fish oil supplements; and a low diet quality score $(<70 \%$ of maximum score) on a 13-item dietary screening tool developed for this study, assessing compliance with Australian dietary guidelines. This was to exclude people already consuming a healthy diet and therefore with less room for improvement in diet quality. Eligible participants were provided with detailed study information and consent form via post or email, and subsequently scheduled to attend a baseline appointment upon providing study 


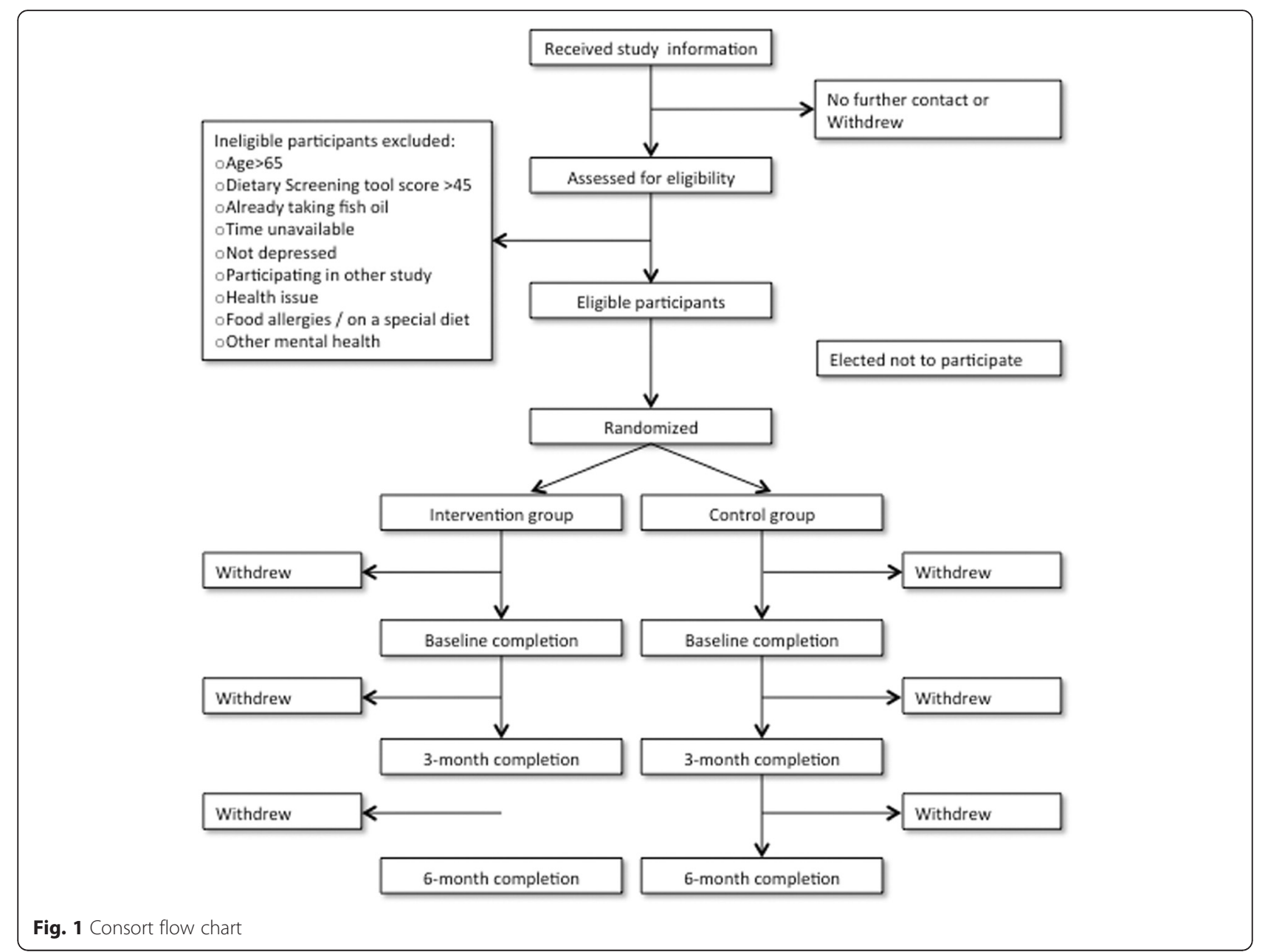

consent. If participants were receiving treatment for their depressive symptoms they were asked to continue what they were doing. Participants who had just started a new treatment protocol at recruitment (anti-depressant medication and/or counselling or psychotherapy); changed their treatment or were intending to change their treatment were delayed in beginning their study participation until they had been undergoing their new treatment protocol for a month. Participants were asked not to start any new treatment or make other lifestyle changes during the study. Prior to attending baseline assessments, participants were randomised by a team member not involved in screening or data collection using the process of minimisation [33], blocked on age and gender, to the intervention or control group. Data collectors and participants were blinded to the treatment allocation until after baseline assessments were completed. Team members conducting data analysis were not involved in data collection, and treatments are blinded until after data are analysed.

All participants attended the Sansom Institute for Health Research clinic at the City East campus of the
University of South Australia at baseline, 3 and 6 months for assessments following an overnight fast (water allowed) for blood and urine samples, blood pressure and anthropometric measurements. They were then provided with a light breakfast and asked to complete questionnaires. Following each round of assessments, participants were given a 3-day food diary and instructions on how to complete it. They were provided with a reply-paid envelope to return the food diaries on completion. All assessments were recorded in individual Case Report Forms according to Good Clinical Practice guidelines (Declaration of Helsinki).

\section{Intervention protocol}

For the first three months of the study, the intervention group received a nutrition education workshop and then six fortnightly group cooking classes, supplemented by four shopping and budgeting education activities during cooking workshops, and recipes and cooking demonstration videos on the HELFIMED.org website. The nutrition education session was led by a nutritionist and dietitian utilising an interactive quiz, PowerPoint presentation on health and the 
Mediterranean diet and food modelling activity based on the Dietary Guidelines for Australians with modifications according to Mediterranean-style dietary principles (i.e. extra virgin olive oil as the principle source of fat; legumes and fish as primary sources of protein). For the cooking workshops, participants worked in small groups of 2-4 to prepare and cook a supplied recipe, within a small workshop setting (2-12 participants) guided by two workshop facilitators. These were conducted in a commercial kitchen at the university, and after preparing the dishes participants ate them together. At each cooking workshop participants received a food hamper that was matched to recipe cooked, containing extra virgin olive oil (donated by Cobram Estate), tinned legumes, tomatoes and tuna (supplied by Simplot), and seasonal fruit, vegetables, and mixed nuts (supplied wholesale by Tony \& Marks Fresh Fruiterers). If participants were unable to attend a cooking workshop in a given fortnight, they were able to collect their food hamper. They were also provided with 2 fish oil capsules per day (grade EPAX 1050 TG each containing $450 \mathrm{mg}$ DHA and 100 EPA per capsule; donated by Epax) for the whole 6 months. The HELFIMED protocol, including nutrition education, cooking workshops and food hampers, was successfully trialed previously in people with serious mental illness [34].

\section{Control group protocol}

The control group attended fortnightly social groups (e.g. sharing holiday stories, playing games, doing personality tests, watching a movie with discussion, book club) during the first three months as a control for the social component of the cooking workshops that can help to improve depressive symptoms. They were provided with free double movie passes as a thank you for completing each round of assessments. On completion of their final round of assessments they were given 3 months' supply of fish oil, a copy of the recipe book provided to the treatment group and attended a nutrition education session.

A clinical Psychologist met with all team members undertaking data collection, cooking workshops and social groups to provide advice and training on how to handle any difficult situations, and was available for referral of participants if counselling was required.

\section{Assessments}

Baseline assessments included fasted blood samples, weight, height, waist circumference and blood pressure, and questionnaires included the Depression Anxiety Stress Scale (DASS-21) [35], Assessment of Quality of Life (AQoL)-8D scale (primary outcomes) [36]; Positive and Negative Affect Scale (PANAS) [37], Simple Dietary Questionnaire (SDQ), Mediterranean diet questionnaire [38] and shopping and budgeting questionnaire. A background questionnaire measured potential covariates: age, gender, socio-economic status, education level $(1=$ completed primary school; $8=$ postgraduate degree), household income, sedentary time and physical activity (each calculated as total minutes per week), sleeping difficulties (how many hours sleep on average on weeknights and weekends; and whether or not they consider sleep to be a problem - yes/no/don't know), smoking status ( $1=$ never smoked; $5=$ smoke daily), and frequency of consuming $>2$ alcoholic drinks per day $(1=$ never/rarely; $5=$ daily). Socioeconomic status was determined from participant postcodes using deciles of the SocioEconomic Indexes For Areas, a ranking of Australian neighbourhood disadvantaged and advantage, based on census data for variables such as education, income and occupation status [39]. Physical activity and sitting time were measured using two questions adapted from the short form of the validated International Physical Activity Questionnaire (IPAQ) [40].

\section{Anthropometric measures}

Height, weight, waist and hip circumference measurements were conducted by trained personnel according to International Standards for Anthropometry and Kinesiology (ISAK) guidelines [41]. Height was measured using a SECA ce0123 portable stadiometer to the nearest $0.1 \mathrm{~cm}$, using the free standing stature technique, whereby the participant's head was aligned in the Frankfort plane and the participant was instructed to take and hold a deep breath while the measurement was taken. Weight was measured by using TANITA ${ }^{\mathrm{m}}$ UM- $021 \mathrm{w}$ calibrated digital scales to the nearest $0.1 \mathrm{~kg}$. Height and weight were used to calculate Body Mass Index $\left(\mathrm{BMI}=\right.$ mass $(\mathrm{kg}) /$ height $\left.\left(\mathrm{m}^{2}\right)\right)$. Waist measurements were taken at the point of the visual narrowing, and hip measurements were taken at the furthest protruding point of the buttocks, using a Luftkin Executive Thinline (W606PM) $2 \mathrm{~m}$ tape. Waist and hip measurements provide a measure of central adiposity, which may be a risk factor for CVD [42]. Two repeat measures were taken for all measurements, and the mean was calculated. If the measurements exceeded $0.5 \mathrm{~cm}$, a third measurement was taken and mean of the three measurements was calculated.

\section{Blood pressure}

Resting blood pressure was measured using an automated Omron sphygmomanometer (Model HEM-7000-C1L). The participant was seated for $5-10$ min prior to taking two measurements, with one minute between measurements. If the difference between the first two measurements exceeded $5 \mathrm{mmHg}$ for either systolic or diastolic pressure, up to two subsequent measurements were taken to obtain two measurements within $5 \mathrm{mmHg}$. 


\section{Blood samples}

Fasting blood samples were collected by trained phlebotomists, and taken to SA Pathology for analysis or spun by low speed centrifugation, aliquoted into $1 \mathrm{ml}$ tubes and stored at $-80{ }^{\circ} \mathrm{C}$ for analysis by specialised laboratories.

Apolipoprotein B (apoB) and AI (apoA1) were measured to assess cardiovascular health. Apolipoproteins are lipid-binding proteins containing cholesterol and lipids. ApoB is a measure of LDL-cholesterol while apoA1 is an indicator of the number of HDL particles. The ratio of apoB/apoA1 has emerged as a strong diagnostic marker of metabolic syndrome [43] and next to smoking as the strongest predictor of cardiovascular disease (specifically risk of myocardial infarction) [44] and was therefore the third primary outcome to assess cardiovascular effects of the intervention. Fasted blood samples were collected in $8 \mathrm{ml}$ serum tubes, and were transported to SA pathology on ice for analysis within two hours of collection.

Omega-3 fatty acids: Fasted blood samples were used to measure erythrocyte levels of n-3 PUFAs in order to assess the direct contribution of fish oil supplementation to any improved outcome measures. Fasted blood samples were collected in $6 \mathrm{ml}$ EDTA tubes, red blood cells were separated from plasma by low speed centrifugation and packed red blood cells were stored in $1 \mathrm{ml}$ micro tubes at $-80 \mathrm{C}$ until analysis. Erythrocyte samples were thawed and prepared for fatty acid analysis according to Swierk et al. [31] using the direct transesterification procedure according to Lepage and Roy [45]. Samples were analysed by flame-ionisation gas chromatography (model GC-17A, Shimadzu) using a $50 \mathrm{~m} \times 0.25 \mathrm{~mm}$ internal diameter capillary column. One microlitre of the sample was auto-injected into the column, and individual fatty acids were quantified using the Shimadzu analysis software (Class-VP 7.2.1 SP1, USA). Fatty acid peaks were identified by comparison with known fatty acid standards and quantitated by comparison to the 21:0 internal standard (Nu-chek and Sigma).

Carotenoids are a group of dietary antioxidant compounds that have been identified as being higher in core components of the Mediterranean diet including certain vegetables, fruit and extra virgin olive oil [46]. These will be measured in blood plasma to provide an objective indicator of increased intake of fruits, vegetables and extra virgin olive oil, and enable correlation with improved outcome measures. Fasting blood samples are collected in evacuated containers with EDTA then wrapped in aluminium foil and kept away from direct light. Centrifugation of vacutainers for $10 \mathrm{~min}(1300 \times \mathrm{g}, 4 \mathrm{oC})$ is done within $1 \mathrm{~h}$ of collection to separate plasma from whole blood. Plasma samples are aliquoted into amber vials and stored at $-80{ }^{\circ} \mathrm{C}$ until analysis. Methods for extraction of carotenoids and preparation of internal standards, external standards, secondary calibrator and plasma samples are based on Karppi et al. [47]. Variations to these methods include the use of argon to evaporate samples prior to reconstitution, and the High Performance Liquid Chromatography (HPLC) model used for analyses is an Agilent 1100 with Kinetex $5 \mu \mathrm{m}$ C18 $100 \AA$ columns $(250 \times 4.6 \mathrm{~mm})$.

Antioxidant and inflammatory markers will be measured in serum. Inflammation and oxidative stress can contribute to the pathophysiology of mood disorders $[4,5,14,48]$ and are associated with diet [48-50]. With blood samples we will investigate the impact of diet on high sensitivity C-Reactive protein, interleukins 1 and 6 , and F2 isoprostane biomarkers and whether these biomarkers mediate any improved outcomes.

\section{Urine samples}

Participants provided a fasted $10 \mathrm{ml}$ urine sample upon arrival, which was taken to SA Pathology for analysis. Urine samples were used to measure the ratio of sodium to potassium intake, which may be related to establish risk factors such as hypertension, obesity and unhealthy eating for cardiovascular diseases and mortality [51-54]. Sodium/potassium ratio can also be an indicator of current food intake, with sodium reflecting intake of processed foods, and potassium reflecting intake of fruit, vegetables and whole foods generally $[52,54-56]$. The cut-off point of less than or equal to one has been recommended [52]. A ratio of one or more may indicate increased risk of high blood pressure [57].

\section{AQoL-8d}

The AQoL-8D is a 35-item questionnaire that is used to measure quality of life. The 35 items load onto eight dimensions, of which three represent physical domains of quality of life (independent living, pain and senses) and five represent psychological domains (happiness, selfworth, coping, relationships, mental health). These load onto two super dimensions (physical and psycho-social). The scores from each dimension are further combined to create a final AQoL-8D utilities score for use in economic evaluation, which will be conducted in this study to measure the cost effectiveness of the program. The AQoL-8D has good validity and internal consistency with alpha coefficients of 0.89-0.96 [36].

\section{DASS-21}

The DASS is a 21-item self-report scale which provides a measure of the level of negative emotional states of depression, anxiety and stress [35]. It is a highly reliable measure that shows high convergent validity and has good internal consistency with a Cronbach alpha of 
$0.82-0.93[58,59]$. To identify degree of severity of these emotional states, DASS-21 sub-scale severity ratings were calculated based on the full DASS-42 severity rating [35] - each scale is multiplied by two and divided into severity categories to yield equivalent scores ranging from 1 (normal) to 5 (extremely severe) for clinical purposes.

\section{PANAS}

The PANAS is a 20 -item scale measuring positive and negative emotions. It has been validated and demonstrates reliable psychometric properties, discriminant, convergent and construct validity. The reliability (internal consistency) of the PANAS positive and negative emotions scales were determined to be $\alpha=.89$ and .85 respectively [37].

\section{Shopping and budgeting questionnaire}

An eighteen-question tailored shopping and budgeting questionnaire was designed to measure knowledge of nutrition, shopping and budgeting. This questionnaire assessed specific topics that were covered in shopping and budgeting education activities conducted during cooking workshops, including knowledge of unit pricing, supermarkets and other food stores (i.e. farmer's markets), smart shopping, prices, budgeting, and reading nutrition labels. This questionnaire will be assessed to determine its psychometric properties.

\section{Dietary questionnaires}

A 14-item Mediterranean diet questionnaire was used to assess level of adherence to the Mediterranean diet. Each item is scored as 0 or 1 , yielding a maximum score of 14 [38]. Higher scores on this questionnaire have been consistently associated with reduced risk of mortality from or incidence of cardiovascular disease [60]. A Simple Dietary Questionnaire (SDQ) measured frequency of intake of a range of food groups including, for the purpose of this study, vegetables, fruit, legumes, wholegrain foods, takeaway foods (examples given were unhealthy options such as burgers, chips, pizza), sweetened drinks, unhealthy sweet and savoury snacks, fish, meat/chicken and nuts. For each question a picture of the food is provided and the portion size is described. For each food group, frequency of intake was reported using six to nine item response scales ranging from never/don't eat to consume seven or more serves per day. For vegetables, fruit, wholegrain foods, takeaway foods, snack foods, nuts, legumes, fish, and meat/chicken a further qualifying question presented a range of different items asking which of those had been consumed in the past two weeks, in order to help prompt memory about foods relevant to the previous item as well as measure dietary variety. For the purpose of this study diversity of fruit and vegetable consumption was measured due to the variety of nutrients and phytonutrients provided by different fruits and vegetables. The SDQ will be validated against 3-day food diaries which participants completed on two week days and one weekend day.

\section{Focus groups}

The intervention group took part in focus groups of three to eight participants at baseline, prior to the nutrition education session, and again at 3 months. The purpose of these was (a) at baseline to identify current nutrition knowledge and food behaviours, enablers and barriers of healthy food consumption, and goals for participation in the program; and (b) at 3 months to identify behavioural changes, whether health goals were achieved, barriers and facilitators to achieving goals, and provide feedback and evaluation of the program. Semi-structured interview guides were used to allow for emerging themes to be explored. Focus groups were recorded, transcribed verbatim and will be analysed using inductive and deductive analysis following the Framework Method [61, 62].

\section{Statistical analysis}

Analyses will be performed using IBM SPSS Version 21.0 for Windows (Chicago, IL, USA) Descriptive statistics using mean and standard deviation or number/percentage for categorical variables will be used to provide baseline demographics and measures, and t-tests or Chi Square will be used to ensure that there are no differences between randomised groups. Empirical distributions will be examined for outcome variables and variables that are not normally distributed will be either transformed or categorised or analysed using nonparametric methods if available. Where assumptions of normality are met, linear mixed modelling will be used to compare changes in the groups over time while adjusting for covariates; this process includes all data therefore allowing analysis on an intent-to-treat basis. To investigate whether changed dietary patterns and omega-3 fatty acid levels were associated with any positive outcomes in mental and/or cardiometabolic health, changes in diet scores, sodium potassium ratio, carotenoids and omega- 3 fatty acids will be entered into correlation or regression analyses as appropriate with changes in outcome scores/markers as dependent variables. Secondary sub-group analyses by depression severity (using DASS categories [35]) will be conducted to determine if the severity of depression has a modification effect on the results. To investigate whether blood antioxidant and inflammatory markers mediate relationships between diet and improved outcomes, pathway models will be fitted to the data with direct and indirect effects tested for their statistical significance. Since these models will involve change over time (growth curve) 
and pathway mechanisms, they will be performed using advanced software tools such as Mplus developed by Muthén and Muthén [63].

\section{Discussion}

Over three months the HELFIMED protocol provided nutrition education and fortnightly cooking workshops and nutrition education based on Mediterranean-style dietary principles which will be followed up at 6 months to investigate the sustainability of any dietary changes, and to explore whether depressive symptoms, mental health, health-related quality of life and cardiometabolic health improved as a result of the intervention. Correlations and/or regression analyses will determine the contributions of dietary changes, carotenoids and omega-3 levels as a result of fish oil consumption to any improved outcomes, and further investigate biochemical markers as potential mediators of these changes over 3 and 6 months. Focus groups and evaluations will identify barriers and enablers of eating healthy diets, and with additional analysis will assist in measuring predictors of retention and improvement to enable further identification of successful program components for adopting a healthy Mediterranean-style diet in people with depression and areas for improvement.

Strengths of the study include the randomisation and controlling for the social component of the cooking workshops and study involvement. The study is limited in that double blinding was not possible and social desirability responses cannot be ruled out. Additionally, the study findings may be limited by using self-report depression at recruitment, however self-report depression has been shown to have a moderate level of agreement with diagnosed depression [32]. This approach was used to maximise recruitment in this population as many individuals with depression do not seek a diagnosis and treatment [64].

This is one of the first randomised controlled trials worldwide to investigate the effect of whole diet, specifically Mediterranean-style diet, on mental health in people with depression. If successful it will support the body of epidemiological data indicating that Mediterranean diet is protective against depression, and will have highly significant implications for treatment and prevention of this prevalent and disabling condition and its substantial impact on the global burden of disease.

\section{Acknowledgments}

We are grateful to all participants who took part in the study; Caitlin Finnis, Thomas Butler, Haruka Ishimoto and Lucy Fairlie-Jones for their assistance with cooking workshops and social groups; Judith Lukas and the School of Pharmacy and Medical Sciences for use of the commercial kitchen; Epax, Pathway International, Cobram Estate, John West, Edgell and Simplot for their generous support with in-kind supply of fish oil capsules and food hamper/cooking workshop items.

\section{Funding}

NP (formerly Sinn), DZ and AW are supported by National Health and Medical Research Council Program Grant funding (\# 320860 and 631947). SBo is supported by Australian Research Council DECRA funding (\# DE130101577). The funding body did not have involvement in the study design, data collection, data analysis and interpretation or preparation of manuscript.

\section{Availability of data and materials}

Not applicable.

\section{Authors' contributions}

NP, DZ, SBo, Cl, LS, KOD participated in study design. DZ, JC, AW, SBo, AV collected data and ran workshops. TN provided statistical advice and input. SBI provided psychological advice and support. LS will oversee economic evaluation. NP and DZ drafted the manuscript. All authors read and approved the final manuscript.

\section{Authors' information}

Not applicable.

\section{Competing interests}

The authors declare that they have no competing interests.

\section{Consent for publication}

Not applicable.

\section{Ethics approval and consent to participate}

Ethics approval was provided by the Human Research Ethics Committee of the University of South Australia (registration no. 0000032674) and all participants provided written informed consent.

\section{Author details}

${ }^{1}$ Centre for Population Health Research, Sansom Institute for Health Research, School of Health Sciences, University of South Australia, Adelaide, Australia. ${ }^{2}$ School of Pharmacy and Medical Sciences, University of South Australia, Adelaide, Australia. ${ }^{3}$ Ehrenberg-Bass Institute for Marketing Science, Business School, University of South Australia, Adelaide, Australia. ${ }^{4}$ Discipline of Dietetics and Human Nutrition, La Trobe University, Melbourne, VIC, Australia. ${ }^{5}$ School of Human Health and Social Sciences, Central Queensland University, Adelaide, South Australia, Australia. ${ }^{6}$ School of Medicine, University of Wollongong, Wollongong, NSW, Australia. ${ }^{7}$ Centre for Population Health Research, School of Health Sciences, University of South Australia, GPO Box 2471, Adelaide, SA 5001, Australia.

Received: 19 March 2016 Accepted: 13 August 2016

Published online: 19 August 2016

References

1. Imamura F, Micha R, Khatibzadeh S, Fahimi S, Shi P, Powles J, Mozaffarian D, on behalf of the Global Burden of Diseases Nutrition and Chronic Diseases Expert Group (NutriCoDE). Dietary quality among men and women in 187 countries in 1990 and 2010: a systematic assessment. Lancet Glob Health. 1990;2015(3):e132-42.

2. Lim SS, Vos T, Flaxman AD, Danaei G, Shibuya K, Adair-Rohani H, AlMazroa MA, Amann M, Anderson HR, Andrews KG, et al. A comparative risk assessment of burden of disease and injury attributable to 67 risk factors and risk factor clusters in 21 regions, 1990-2010. A systematic analysis for the global burden of disease study 2010. Lancet. 2013;2013(380):2224-60.

3. Mathers CD, Loncar D. Projections of global mortality and burden of disease from 2002 to 2030. PLoS Med. 2006;3, e442

4. Gimeno D, Kivimaki M, Brunner EJ, Elovainio M, De Vogli R, Steptoe A, Kumari M, Lowe GDO, Rumley A, Marmot MG, et al. Associations of creactive protein and interleukin- 6 with cognitive symptoms of depression: 12-year follow-up of the whitehall ii study. Psychol Med. 2009;39:413-23.

5. Pasco JA, Nicholson GC, Williams LJ, Jacka FN, Henry MJ, Kotowicz MA, Schneider HG, Leonard BE, Berk M. Association of high-sensitivity c-reactive protein with de novo major depression. Br J Psychiatry. 2010;197:372-7.

6. Joynt KE, Whellan DJ, O'Connor CM. Depression and cardiovascular disease: Mechanisms of interaction. Biol Psychiatry. 2003;54:248-61. 
7. Perez-Cornago A, de la Iglesia R, Lopez-Legarrea P, Abete I, Navas-Carretero S, Lacunza Cl, Lahortiga F, Martinez-Gonzalez MA, Martinez JA, Zulet MA. A decline in inflammation is associated with less depressive symptoms after a dietary intervention in metabolic syndrome patients: a longitudinal study. Nutr J. 2014;13:1.

8. Bach-Faig A, Berry EM, Lairon D, Reguant J, Trichopoulou A, Dernini S, Medina FX, Battino M, Belahsen R, Miranda G, et al. Mediterranean diet pyramid today. Science and cultural updates. Public Health Nutr. 2011;14: 2274-84.

9. Davis C, Bryan J, Hodgson J, Murphy K. Definition of the Mediterranean diet: a literature review. Nutrients. 2015;7:9139-53.

10. Grosso G, Marventano S, Yang J, Micek A, Pajak A, Scalfi L, Galvano F, Kales $\mathrm{SN}$. A comprehensive meta-analysis on evidence of Mediterranean diet and cardiovascular disease: Are individual components equal? Crit Rev Food Sci Nutr. 2015. doi:10.1080/10408398.2015.1107021.

11. Sofi F, Cesari F, Abbate R, Gensini GF, Casini A. Adherence to Mediterranean diet and health status: a meta-analysis. BMJ. 2008;337:a1344.

12. Estruch R, Ros E, Salas-Salvado J, Covas M-I, Corella D, Aros F, Gomez-Gracia E, Ruiz-Gutierrez V, Fiol M, Lapetra J, et al. Primary prevention of cardiovascular disease with a mediterranean diet. N Engl J Med. 2013;368:1279-90.

13. Nordmann AJ, Suter-Zimmermann K, Bucher HC, Shai I, Tuttle KR, Estruch R, Briel M. Meta-analysis comparing mediterranean to low-fat diets for modification of cardiovascular risk factors. Am J Med. 2011;124:841-51.

14. Parletta N, Milte CM, Meyer B. Nutritional modulation of cognitive function and mental health. J Nutr Biochem. 2013;24:725-43.

15. Freeman MP. Omega-3 fatty acids in psychiatry: a review. Ann Clin Psychiatry. 2000;12:159-65.

16. Sinn N, Milte C, Howe PRC. Oiling the brain: a review of randomised controlled trials of omega-3 fatty acids in psychopathology across the lifespan. Nutrients. 2010;2:128-70.

17. Lai JS, Hiles S, Bisquera A, Hure AJ, McEvoy M, Attia J. A systematic review and meta-analysis of dietary patterns and depression in community-dwelling adults. Am J Clin Nutr. 2014;99:181-97.

18. Psaltopoulou T, Sergentanis TN, Panagiotakos DB, Sergentanis IN, Kosti R, Scarmeas N. Mediterranean diet, stroke, cognitive impairment, and depression: a meta-analysis. Ann Neurol. 2013;74:580-91.

19. Freeman MP. Nutrition and psychiatry. Am J Psychiatry. 2010;167:244-7.

20. Jacka FN, Mykletun A, Berk M. Moving towards a population health approach to the primary prevention of common mental disorders. BMC Med. 2012;10:149.

21. Zazpe I, Sanchez-Tainta A, Estruch R, Lamuela-Raventos RM, Schröder $H$, Salas-Salvado J, Corella D, Fiol M, Gomez-Gracia E, Aros F, et al. A large randomised individual and group intervention conducted by registered dietitians increased adherence to Mediterranean-type diets: the predimed study. J Am Diet Assoc. 2008;108:1134-44

22. Sanchez-Villegas, A.; Martínez-Gonzalez, M.A.; Estruch, R.; Salas-Salvadó, J.; Corella, D.; Covas, M.-I.; Aromaa, A.; Arós, F.; Romaguera, D.; Gómez-Gracia, E., et al. Mediterranean dietary pattern and depression: The predimed randomized trial. BMC Medicine 2013, 11, doi:10.1186/1741-7015-1111-1208

23. Perez-Cornago A, Lopez-Legarrea $P$, de la Iglesia $R$, Lahortiga F, Martinez JA, Zulet MA. Longitudinal relationship of diet and oxidative stress with depressive symptoms in patients with metabolic syndrome after following a weight loss treatment: the resmena project. Clin Nutr. 2014;33:1061-7.

24. Stahl ST, Albert SM, Dew MA, Lockovich MH, Reynolds CF. Coaching in healthy dietary practices in at-risk older adults: a case of indicated depression prevention. Am J Psychiatry. 2014;171:499-505.

25. O'Neil A, Berk M, Itsiopoulos C, Castle D, Opie R, Pizzinga J, Brazionis L, Hodge A, Mihalopoulos C, Chatterton ML, et al. A randomised, controlled trial of a dietary intervention for adults with major depression (the "smiles" trial): study protocol. BMC Psychiatry. 2013;13:114.

26. Barth J, Schumacher M, Herrmann-Lingen C. Depression as a risk factor for mortality in patients with coronary heart disease: a meta-analysis. Psychosom Med. 2004;66:802-13.

27. Simopoulos AP. The importance of the omega-6/omega-3 fatty acid ratio in cardiovascular disease and other chronic diseases. Exp Biol Med. 2008;233:674-88.

28. Harris WS, von Schacky C. The omega-3 index: a new risk factor for death from coronary heart disease? Prev Med. 2004;39:212-20.

29. Parletta N, Zarnowiecki D, Cho J, Wilson A, Procter N, Gordon A Bogomolova S, O'Dea K, Strachan J, Ballestrin M. People with schizophrenia and depression have a low omega-3 index. Prostaglandins Leukot Essent Fatty Acids. 2016;110:42-7.
30. Sullivan BL, Williams PG, Meyer BJ. Biomarker validation of a long-chain omega-3 polyunsaturated fatty acid food frequency questionnaire. Lipids. 2006;41:845-50.

31. Swierk M, Williams PG, Wilcox J, Russell KG, Meyer BJ. Validation of an australian electronic food frequency questionnaire to measure polyunsaturated fatty acid intake. Nutrition. 2011;27:641-6.

32. Stuart AL, Pasco JA, Jacka FN, Brennan SL, Berk M, Williams LJ. Comparison of self-report and structured clinical interview in the identification of depression. Compr Psychiatry. 2014;55:866-9.

33. Altman DG, Bland JM. Treatment allocation by minimisation. Br Med J. 2005:330:843.

34. Bogomolova S, Zarnowiecki D, Wilson A, Fielder A, Procter N, Itsiopoulos C, O'Dea K, Strachan J, Ballestrin M, Champion A, Parletta N, Dietary intervention for people with mental illness in South Australia. Health Promotion International:daw055.

35. Lovibond SH, Lovibond PF. Manual for the depression anxiety stress scales. 2nd ed. Sydney: Psychology Foundation; 1995.

36. Richardson J, lezzi A, Khan MA, Maxwell A. Validity and reliability of the assessment of quality of life (aqol)-8d multi-attribute utility instrument. Patient. 2014;7:85-96.

37. Crawford JR, Henry JD. The positive and negative affect schedule (panas): construct validity, measurement properties and normative data in a large non-clinical sample. Br J Clin Psychol. 2004:43:245-65.

38. Martinez-Gonzalez MA, Garcia-Arellano A, Toledo E, Salas-Salvado J, BuilCosiales P, Corella D, Covas M-I, Schroder H, Aros F, Gomez-Gracia E, et al. A 14-item Mediterranean diet assessment tool and obesity indexes among high-risk subjects: the predimed trial. PLoS One 2012:7, e43134.

39. Pink B. Socio-economic indexes for areas (seifa). Canberra: Australian Bureau of Statistics; 2011

40. Booth ML, Ainsworth BE, Pratt M, Ekelund U, Yngve A, Sallis JF, Oja P. International physical activity questionnaire: 12-country reliability and validity. Med Sci Sports Exerc. 2003;195:1381-95

41. Marfell-Jones M, Stewart AD, de Ridder JH. International standards for anthropometric assessment. International Society for the Advancement of Kinanthropometry. New Zealand: Wellington; 2012

42. Han TS, Van EM, Seidell JC, Lean EJ. Waist circumference action levels in the identification of cardiovascular risk factors: Prevalence study in a random sample. Br Med J. 1995;311:1401-5.

43. Pitsavos C, Panagiotakos DB, Skoumas J, Papadimitriou L, Stefanadis C. Risk stratification of apolipoprotein b, apolipoprotein ai, and apolipoprotein b/ai ratio on the prevalence of the metabolic syndrome: the Attica study. Angiology. 2008:59:335-41.

44. Yusuf S, Hawken S, Ôunpuu S, Dans T, Avezum A, Lanas F, McQueen M, Budaj A, Pais P, Varigos J, et al. Effect of potentially modifiable risk factors associated with myocardial infarction in 52 countries (the interheart study): case-control study. Lancet. 2004;364:937-52.

45. Lepage G, Roy CC. Direct transesterification of all classes of lipids in a one-step reaction. J Lipid Res. 1986:27:114-20.

46. Su Q, Rowley KG, Itsiopoulos C, O'Dea K. Identification and quantitation of major carotenoids in selected components of the Mediterranean diet: green leafy vegetables, figs and olive oil. Eur J Clin Nutr. 2002;56:1149-54.

47. Karppi J, Nurmi T, Olmedilla-Alonso B, Granado-Lorencio F, Nyyssönen K. Simultaneous measurement of retinol, a-tocopherol and six carotenoids in human plasma by using an isocratic reversed-phase hplc method. J Chromatogr B. 2008;867:226-32.

48. Ng F, Berk M, Dean O, Bush Al. Oxidative stress in psychiatric disorders: evidence base and therapeutic implications. Int J Neuropsychopharmacol. 2008;21:1-26 [Epub ahead of print].

49. Cavicchia PP, Steck SE, Hurley TG, Hussey JR, Ma Y, Ockene IS, Hébert JR. A new dietary inflammatory index predicts interval changes in serum high-sensitivity c-reactive protein. J Nutr. 2009;139:2365-72.

50. Shivappa N, Steck SE, Hurley TG, Hussey JR, Ma Y, Ockene IS, Tabung F, Hébert JR. A population-based dietary inflammatory index predicts levels of c-reactive protein in the seasonal variation of blood cholesterol study (seasons). Public Health Nutr. 2014;17:1825-33.

51. Cogswell ME, Elliott P, Wang CY, Rhodes DG, Pfeiffer CM, Loria CM. Assessing U.S. Sodium intake through dietary data and urine biomarkers. Adv Nutr. 2013;4:560-2.

52. Drewnowski A, Maillot M, Rehm C. Reducing the sodium-potassium ratio in the us diet: a challenge for public health. Am J Clin Nutr. 2012;96:439-44. 
53. Huggins CE, O'Reilly S, Brinkman M, Hodge A, Giles GG, English DR, Newson CA. Relationship of urinary sodium and sodium-to-potassium ratio to blood pressure in older adults in australia. Med J Aust. 2011;195:128-32.

54. Judd SE, Aaron KJ, Letter AJ, Jenny NS, Campbell RC, Kabagambe EK, Levitan EB, Levine DA, Shikany JM, Safford M, et al. High sodium: potassium intake ratio increases the risk for all-cause mortality: the reasons for geographic and racial differences in stroke (regards) study. J Nutr Sci. 2013;2, e13.

55. Meneton P, Lafay L, Tard A, Dufour A, Ireland J, Ménard J, Volatier JL. Dietary sources and correlates of sodium and potassium intakes in the french general population. Eur J Clin Nutr. 2009;63:1169-75.

56. Yang Q, Liu T, Kuklina EV, Flanders WD, Hong Y, Gillespie C, Chang M-H, Gwinn M, Dowling N, Khoury MJ. Sodium and potassium intake and mortality among us adults: prospective data from the third national health and nutrition examination survey. Arch Intern Med. 2011;171:1183-91.

57. Stanton JL, Braitman LE, Riley A, Khoo C-S, Smith JL. Demographic, dietary, life style, and anthropometric correlates of blood pressure. Hypertension. 1982;4, III135.

58. Crawford JR, Cayley C, Lovibond PF, Wilson PH, Hartley C. Percentile norms and accompanying interval estimates from an australian general adult population sample for self-report mood scales (bai, bdi, crsd, ces-d, dass, dass-21, stai-x, stai-y, srds, and sras). Aust Psychol. 2011;46:3-14.

59. Henry JD, Crawford JR. The short-form version of the depression anxiety stress scales (dass-21): construct validity and normative data in a large non-clinical sample. Br J Clin Psychol. 2005;44:227-39.

60. Martinez-Gonzalez MA, Bes-Rastrollo M. Dietary patterns, mediterranean diet, and cardiovascular disease. Curr Opin Lipidol. 2014:25:20-6.

61. Gale NK, Heath G, Cameron E, Rashid S, Redwood S. Using the framework method for the analysis of qualitative data in multi-disciplinary health research. BMC Med Res Methodol. 2013;13.

62. Smith J, Firth J. Qualitative data analysis: the framework approach. Nurse Res. 2011;18:52-62.

63. Muthén, L.K.; Muthén, B.O. Mplus user's guide, seventh edition. Muthén \& Muthén: Los Angeles, CA, 1998-2012

64. Barney $\sqcup$, Griffiths KM, Jorm AF, Christensen H. Stigma about depression and its impact on help-seeking intentions. Aust N Z J Psychiatry. 2006:40:51-4.

\section{Submit your next manuscript to BioMed Central and we will help you at every step:}

- We accept pre-submission inquiries

- Our selector tool helps you to find the most relevant journal

- We provide round the clock customer support

- Convenient online submission

- Thorough peer review

- Inclusion in PubMed and all major indexing services

- Maximum visibility for your research

Submit your manuscript at www.biomedcentral.com/submit

) Biomed Central 